\title{
Performance improvement of polycrystalline diamond ultraviolet photodetectors by room-temperature plasma treatment
}

\author{
S. G. Wang, ${ }^{\text {a) }}$ P. J. Sellin, and A. Lohstroh \\ Department of Physics, School of Electronics and Physical Sciences, University of Surrey, \\ Guildford GU2 7XH, United Kingdom \\ Qing Zhang \\ Microelectronics Centre, Nanyang Technological University, Nanyang Avenue, Singapore 639798, Singapore
}

(Received 7 September 2004; accepted 4 January 2005; published online 23 February 2005)

\begin{abstract}
Enhancement of ultraviolet (UV) photoresponsivity in chemical-vapor-deposited (CVD) diamond photodetectors was observed by posttreatment in a plasma of oxygen and carbon tetrafluoride at room temperature. This room-temperature plasma posttreatment was found to be an efficient process in suppressing the extrinsic photoresponse of CVD diamond UV photodetectors in the visible region. Nearly four orders of magnitude difference in the photoresponsivity between the UV and visible light regions were obtained. The results of photoluminescence mapping and Raman spectra indicate that this performance improvement may have resulted from the effective removal of the $s p^{2}$-bonded carbon impurities and passivation of the silicon-vacancy defects in the diamond. (C) 2005 American Institute of Physics. [DOI: 10.1063/1.1868862]
\end{abstract}

Diamond, which is intrinsically visible blind due to its wide band gap of $5.5 \mathrm{eV}$, is an ideal candidate for ultraviolet (UV) photodetector applications. ${ }^{1}$ However, an appreciable amount of nondiamond phase impurities and structural defects commonly exists in chemical-vapor-deposited (CVD) diamond which significantly enhances the extrinsic photoresponse in the visible region, dramatically deteriorating the performance of the diamond for UV photodetection. ${ }^{2}$ Many attempts have been made to reduce the photoresponsivity in the visible region, including device design and material treatments. Among the various material treatments, McKeag et $a l .^{3}$ demonstrated that a methane-air treatment at a high temperature of $700{ }^{\circ} \mathrm{C}$ can lead to a virtual extinction of any extrinsic photoresponse, greatly decreasing the photoresponsivity of diamond photodetectors in the visible light region, but the substitution of either gaseous environment for inert species such as argon or nitrogen did not produce the same effect. On the other hand, plasma posttreatment at room temperature to efficiently improve the performance of diamond UV photodetectors has not been reported. In this letter, we report a room-temperature post deposition process applied to CVD diamond samples. This process, which employed a plasma of oxygen and carbon tetrafluoride, was simple in operation and low cost. This resulted in an improved UV photoresponse at $225 \mathrm{~nm}$, by nearly four orders of magnitude with respect to the visible wavelength.

The CVD diamond samples used in this study were grown on silicon substrates in a microwave plasma-enhanced CVD (MPECVD) reactor $(1.5 \mathrm{~kW}$ and $2.45 \mathrm{GHz})$ using $1 \%$ methane diluted in hydrogen as a feed gas and with a substrate temperature of $720^{\circ} \mathrm{C}$. The as-grown diamond films had a thickness of $20 \mu \mathrm{m}$ and a polycrystalline structure with a mean grain size of $14 \mu \mathrm{m}$ at the growth surface. Photolithography was used to form an interdigitated planar array of $25 \mu \mathrm{m}$ wide gold contacts, with a $25 \mu \mathrm{m}$ interstrip spacing on the top surface of the diamond samples. Some of the photodetectors were then subjected to a treatment in the

\footnotetext{
${ }^{a)}$ Electronic mail: s.wang@surrey.ac.uk
}

plasma of oxygen and carbon tetrafluoride at room temperature $\left(23 \pm 2{ }^{\circ} \mathrm{C}\right)$. The process conditions were a pressure of 30 mTorr, a radio frequency (rf) power $(13.56 \mathrm{MHz})$ of $300 \mathrm{~W}$ and a reaction gas mixture of $90 \%$ carbon tetrafluoride and $10 \%$ oxygen. This was performed in a commercial reactive ion etching system for half an hour. The UV-visible photoconductive responsivity of the detectors was investigated by exposing the devices to monochromatic light. UV illumination was supplied with a deuterium lamp $(180-350 \mathrm{~nm})$ and visible light illumination was provided using a Xe arc lamp (350-700 nm). The photocurrent was measured using a Keithley 237 source/measurement unit. The incident optical power spectrum was measured using a precalibrated UV-enhanced silicon photodiode.

The photoresponsivity of the diamond UV photodetectors against the wavelength of the incident light is shown in Fig. 1. Interestingly, the detectors that were subjected to the room-temperature plasma posttreatment show a sharp increased response in the UV region peaking at $225 \mathrm{~nm}$, which corresponds to the diamond band-to-band transition, giving rise to nearly four orders of magnitude higher response than that seen in the visible region.

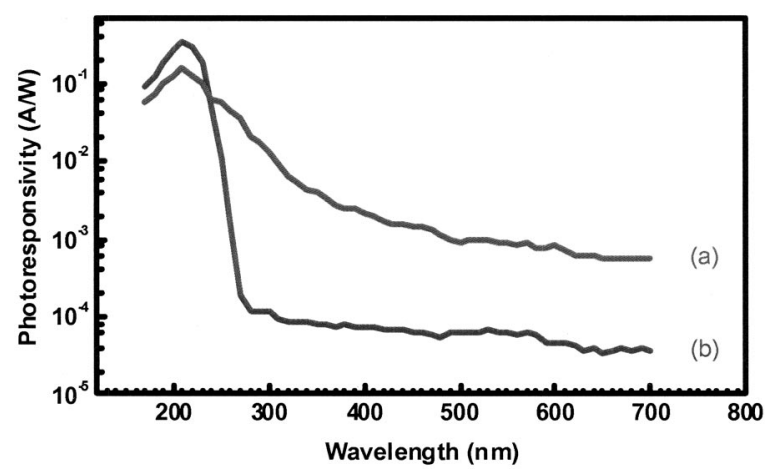

FIG. 1. Photoresponsivity of CVD diamond photodetectors (a) before and (b) after room-temperature plasma treatment. 


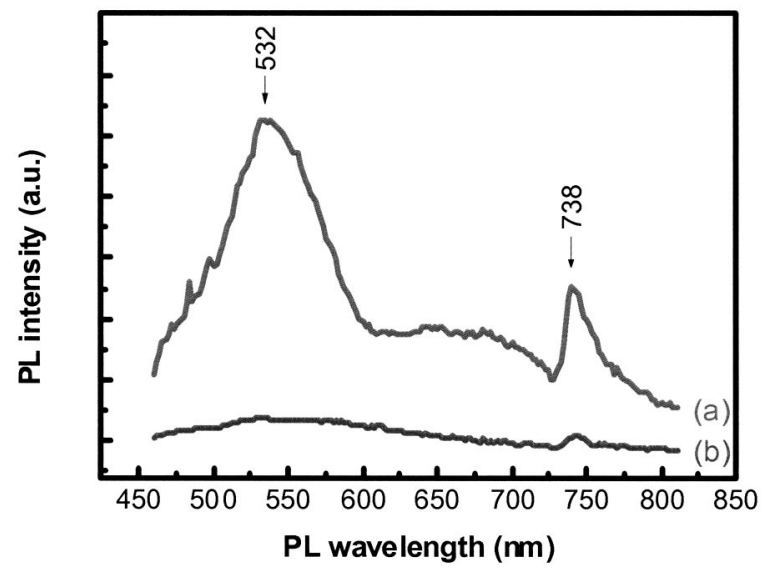

FIG. 2. Room-temperature PL spectra for CVD diamond samples (a) before and (b) after room-temperature plasma treatment.

The room-temperature PL spectra from both the plasma posttreated and the as-grown untreated CVD diamond samples are displayed in Fig. 2. A $20 \mathrm{~mW}$ violet semiconductor laser $(\lambda=405 \pm 3 \mathrm{~nm})$ was used as an excitation source. Two PL bands centered at $738 \mathrm{~nm}(1.68 \mathrm{eV})$ and $532 \mathrm{~nm}(2.33 \mathrm{eV})$ were observed from the untreated sample, see Fig. 2(a). In the plasma posttreated sample the peak at $532 \mathrm{~nm}(2.33 \mathrm{eV})$ disappeared and only one PL band centered at $738 \mathrm{~nm}(1.68 \mathrm{eV})$ was detected, as shown in Fig. 2(b). The presence of $s p^{2}$-bonded carbon phases is believed to be responsible for the band centered at $532 \mathrm{~nm}(2.33 \mathrm{eV}){ }^{4}$ The results presented in Fig. 2(b) suggest that the plasma posttreatment causes etching of the $s p^{2}$-bonded carbon impurities in the diamond. The PL band centered at $738 \mathrm{~nm}$ $(1.68 \mathrm{eV})$ is attributed to a silicon-vacancy defect complex $(\mathrm{Si}-\mathrm{V}) .^{5}$ The unintentional incorporation of the extrinsic silicon in our diamond film is assumed to result mainly from the interface diffusion of silicon atoms during nucleation and growth from the silicon substrate. In Fig. 2(b), one can observe that the intensity of the silicon-vacancy complex line decreases after plasma treatment. It was not possible to determine the absolute concentration of impurities and defects in the samples from the PL spectra. In order to examine the distribution of impurities or defect centers, room-temperature PL mapping was performed for the samples before and after posttreatment. Figure 3 shows the PL mapping images obtained using the same violet laser. The $50 \times 50$ pixels correspond to a mapping area of $5 \times 5 \mathrm{~mm}^{2}$. Bright pixels in the images mark the areas of high luminescent intensity at the stated wavelength. Figure 3 indicates that the luminescent bands both at $532 \mathrm{~nm}(2.33 \mathrm{eV})$ and $738 \mathrm{~nm}(1.68 \mathrm{eV}) \mathrm{de}-$ creased dramatically after plasma posttreatment. This reveals that the room-temperature plasma oxidation posttreatment can remove the $s p^{2}$-bonded carbon and passivate the siliconvacancy defects.

Figure 4 presents the Raman spectra of CVD diamond samples before and after the room-temperature plasma treatment. The Raman spectra were recorded employing a Renishaw micro-Raman spectrometer using an $\mathrm{Ar}^{+}$laser $(\lambda$ $=514.5 \mathrm{~nm}$ ) as the excitation source. A sharp diamond line at $1332 \mathrm{~cm}^{-1}$ along with a weak broad amorphous carbon peak at around $1550 \mathrm{~cm}^{-1}$ was detected in the sample before plasma treatment. No features of $s p^{2}$-bonded carbon phases but only a sharp diamond line at $1332 \mathrm{~cm}^{-1}$ were detected in Downloaded 30 Mar 2009 to 131.227.178.132. Redistribution subject

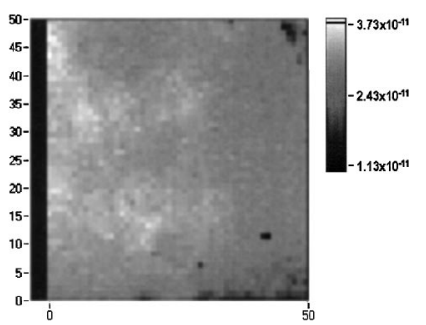

(a)

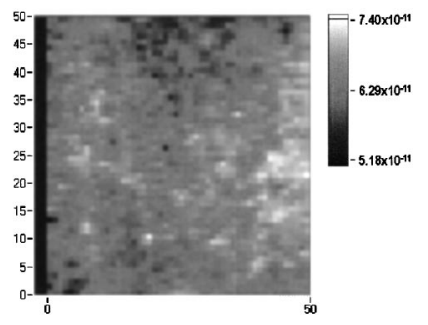

(c)

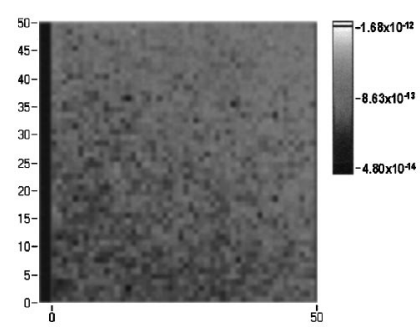

(b)

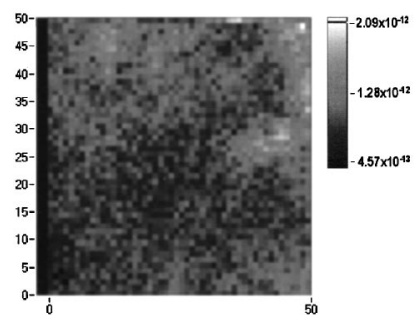

(d)
FIG. 3. Room-temperature PL mapping images. Each map shows the PL intensities at a particular wavelength: (a) $532 \mathrm{~nm}$ before, (b) $532 \mathrm{~nm}$ after, (c) $738 \mathrm{~nm}$ before, and (d) $738 \mathrm{~nm}$ after plasma treatment for CVD diamond samples, respectively.

the plasma posttreated sample. However, the background of the Raman spectrum decreased dramatically in the posttreated sample. The full-width at half-maximum (FWHM) value of the $1332 \mathrm{~cm}^{-1}$ line also decreased from 4.8 to $3.2 \mathrm{~cm}^{-1}$ after plasma treatment. The decrease in FWHM and the background is possibly caused by the removal of the $s p^{2}$-bonded carbon impurities and defect passivation in the diamond surface regions, in good agreement with the PL characterization results.

Photoresponsivity for the band gap less than $5.5 \mathrm{eV}$ is due either to the generation of free electrons into the conduction band from deep defect centers situated in the band gap, or to the generation of free holes in the valence band, resulting from the excitation of electrons from the valence band to deep trapping centers. The deep levels in the diamond band gap are related to impurities and defects. In the case of irradiation at photon energies of more than $5.5 \mathrm{eV}$ $(\lambda<225 \mathrm{~nm})$, the direct excitation of electrons from the valence band to the conduction band, intrinsic excitation oc-

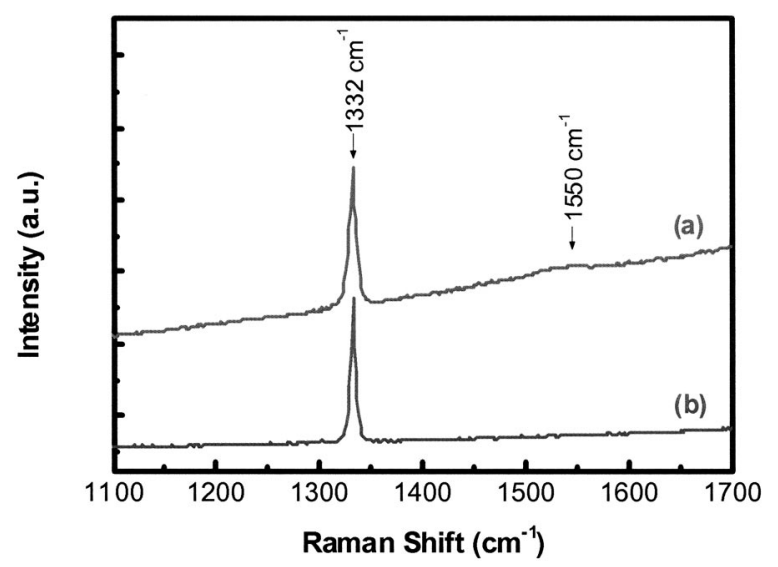

FIG. 4. Raman spectra for CVD diamond (a) before and (b) after roomtemperature plasma treatment.

to AIP license or copyright; see http://apl.aip.org/apl/copyright.jsp 
curs. On the other hand, the impurities can also play a role in the recombination of photogenerated carriers. The Raman spectra combined with the PL mapping results from the plasma-treated samples clearly indicate the decrease in $s p^{2}$-bonded carbon impurities and silicon-vacancy defect centers which is believed to reduce the subgap photoresponse, and hence increase the collection efficiency in the band-to-band excitation, resulting in an increase of photoresponsivity in the UV light region and a decrease in the visible light region. Further work is in progress to clarify the detailed mechanism.

In conclusion, CVD diamond UV photodetectors were characterized by their UV photoresponsivity. The posttreatment process in the plasma of oxygen and carbon tetrafluoride at room temperature described here, was found to be a very effective way of eliminating the $s p^{2}$-bonded carbon impurities and passivating the silicon-vacancy defects. This resulted in removing the visible component of the photoresponse and therefore significantly enhancing the UV photoresponse of our devices.

1P. J. Dean, E. C. Lightowlers, and D. R. Wight, Phys. Rev. A 140, 352 (1965).

${ }^{2}$ L. S. Pan, D. R. Kania, S. Han, J. W. Auger, M. Landstrass, O. L. Landen, and P. Pianetta, Science 255, 830 (1992).

${ }^{3}$ R. D. McKeag, S. S. M. Chan, and R. B. Jackman, Appl. Phys. Lett. 67, 2117 (1995).

${ }^{4}$ M. Nesladek, L. M. Stals, and A. Stesmans, Appl. Phys. Lett. 72, 3306 (1996).

5J. Ruan, W. J. Choyke, and W. D. Partlow, Appl. Phys. Lett. 58, 295 (1991). 